\title{
Sonographic tracking of trunk nerves: essential for ultrasound-guided pain management and research
}

This article was published in the following Dove Press journal:

Journal of Pain Research

4 January 2017

Number of times this article has been viewed

\author{
Ke-Vin Chang ${ }^{1,2}$ \\ Chih-Peng $\operatorname{Lin}^{2,3}$ \\ Chia-Shiang $\operatorname{Lin}^{4,5}$ \\ Wei-Ting Wu' \\ Manoj K Karmakar ${ }^{6}$ \\ Levent Özçakar ${ }^{7}$
}

'Department of Physical Medicine and Rehabilitation, National Taiwan University Hospital, Bei-Hu Branch, Taipei, Taiwan; ${ }^{2}$ National Taiwan University College of Medicine, Taipei, Taiwan; ${ }^{3}$ Department of Anesthesiology, National Taiwan University Hospital, Taipei, Taiwan; ${ }^{4}$ Department of Anesthesiology, Mackay Memorial Hospital, Taipei, Taiwan; ${ }^{5}$ Mackay Medicine, Nursing and Management College, Mackay Medical College, Taipei, Taiwan; ${ }^{6}$ Department of Anesthesia and Intensive Care, Chinese University of Hong Kong, Prince of Wales Hospital, New Territories, Hong Kong; ${ }^{7}$ Department of Physical and Rehabilitation Medicine, Hacettepe University Medical School, Ankara, Turkey
Correspondence: Chih-Peng Lin Department of Anesthesiology, National Taiwan University Hospital, No. 7, Chung Shan S Rd, Zhongzheng Dist, Taipei City 10002, Taiwan

Email cplin0I23@gmail.com

\begin{abstract}
Delineation of architecture of peripheral nerves can be successfully achieved by high-resolution ultrasound (US), which is essential for US-guided pain management. There are numerous musculoskeletal pain syndromes involving the trunk nerves necessitating US for evaluation and guided interventions. The most common peripheral nerve disorders at the trunk region include thoracic outlet syndrome (brachial plexus), scapular winging (long thoracic nerve), interscapular pain (dorsal scapular nerve), and lumbar facet joint syndrome (medial branches of spinal nerves). Until now, there is no single article systematically summarizing the anatomy, sonographic pictures, and video demonstration of scanning techniques regarding trunk nerves. In this review, the authors have incorporated serial figures of transducer placement, US images, and videos for scanning the nerves in the trunk region and hope this paper helps physicians familiarize themselves with nerve sonoanatomy and further apply this technique for US-guided pain medicine and research.
\end{abstract}

Keywords: ultrasound, nerve, trunk, lumbar, pain

\section{Introduction}

Delineation of peripheral nerve architecture can be successfully achieved by highresolution ultrasound (US), which is essential for US-guided pain management and research. Recently, review articles incorporating US images and video demonstrations have been published as regards neck and the upper/lower extremities. ${ }^{1-3}$ Also, there are numerous musculoskeletal pain syndromes related to nerve disorders necessitating US imaging for evaluation and guided interventions in the trunk area.

For instance, the costoclavicular junction is the narrowest portion of the brachial plexus passage, requiring delicate examination in patients presenting with thoracic outlet syndrome. ${ }^{4}$ Scapular winging indicates the scapulae protruding out of the rib cage and is caused by serratus anterior paralysis and needs assessment of the long thoracic nerve from its cervical origin to the lateral thoracic attachment. ${ }^{5}$ Treating interscapular pain is challenging, and injection to the dorsal scapular nerve over the upper back can be helpful for pain relief. ${ }^{6}$ Lumbar facet syndrome is a common cause of low back pain that can be effectively managed by blocking the medial branches of lumbar spinal nerves. ${ }^{7,8}$ Therefore, familiarization of scanning techniques over the trunk nerves is noteworthy for physicians concerning the diagnoses of several neuromuscular problems and relevant therapeutic interventions. Accordingly, the present review incorporates serial figures of transducer placement, US images, and videos for scanning the nerves in the trunk region, and with this the authors hope 
to help physicians familiarize with nerve sonoanatomy and further apply this technique in US-guided pain medicine and research.

\section{Infraclavicular brachial plexus Anatomy}

The brachial plexus can be divided into five levels from its proximal origins to distal ends including the roots, trunks, divisions, cords, and peripheral branches. The medial, posterior, and lateral cords, which are named according to their positions in relation to the axillary artery, are located at the infraclavicular region. At its most lateral part is the retropectoralis minor space, which is bordered by the pectoralis minor muscle anteriorly, subscapularis muscle superior and posteriorly, and the anterior chest wall inferior and posteriorly. ${ }^{9,10}$

\section{Technique}

The transducer is placed in the sagittal plane caudal to the clavicle (Figure 1A) and moved inferior and lateral to align with the pectoralis minor muscle (Figure 1B). The brachial plexus is seen surrounding the axillary artery underneath the pectoralis minor muscle. The lateral, posterior, and medial cords course lateral, deep, and medial to the axillary artery, respectively. The transducer can also be pivoted to visualize the cords in their long axis view (Figure 1C and D). The brachial plexus usually gives off their terminal branches at the lateral edge of the pectoralis minor muscle (Video S1).

\section{Long thoracic nerve Anatomy}

The long thoracic nerve arises from the ventral rami of the fifth to seventh cervical nerves and then passes underneath the clavicle to reach the lateral aspect of the thoracic wall. It courses along the midaxillary line and above the serratus anterior muscle. It is accompanied by the lateral thoracic artery, a branch of the subclavian artery, and innervates the serratus anterior muscle. ${ }^{11}$

\section{Technique}

During arm abduction, the transducer is placed on the midaxillary line in the horizontal plane. The nerve is seen emerging from the junction between the pectoralis major and serratus anterior muscles (Figure 2A). Using the power Doppler mode, the lateral thoracic artery can be localized. The long thoracic nerve is seen as a hypoechoic ovoid structure near the artery. Moving the transducer caudally, visualization of the nerve coursing above the serratus anterior muscle can be possible (Figure 2B and Video S2).
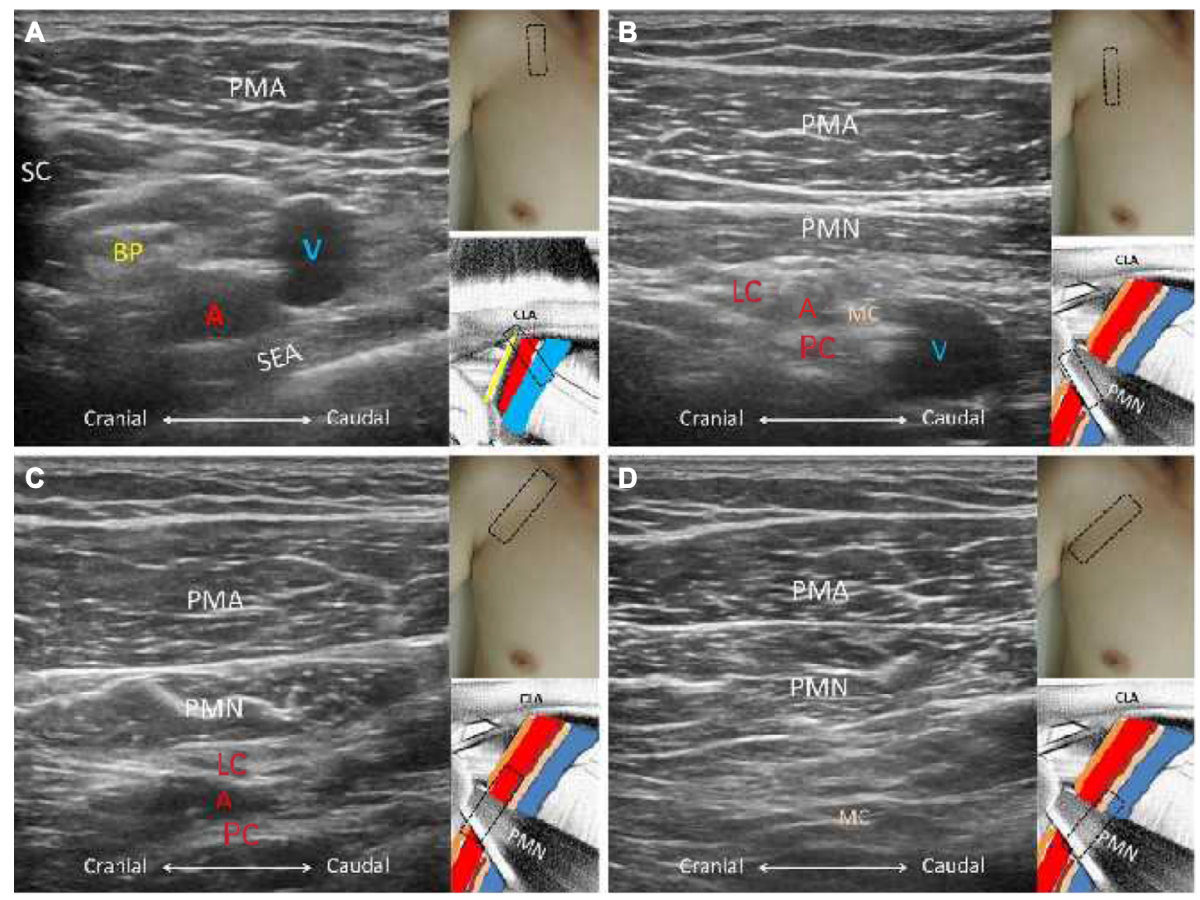

Figure I The short axis views of the brachial plexus just distal to the clavicle (A) and underneath the pectoralis minor muscle (B); the long axis views of the lateral and posterior cords (C) and the medial cord (D).

Note: The red A indicates axillary artery.

Abbreviations: BP, brachial plexus; LC, lateral cord; PC, posterior cord; MC, medial cord; PMA, pectoralis major; PMN, pectoralis minor; SC, subclavius; SEA, serratus anterior; $\mathrm{V}$, axillary vein; CLA, clavicle. 

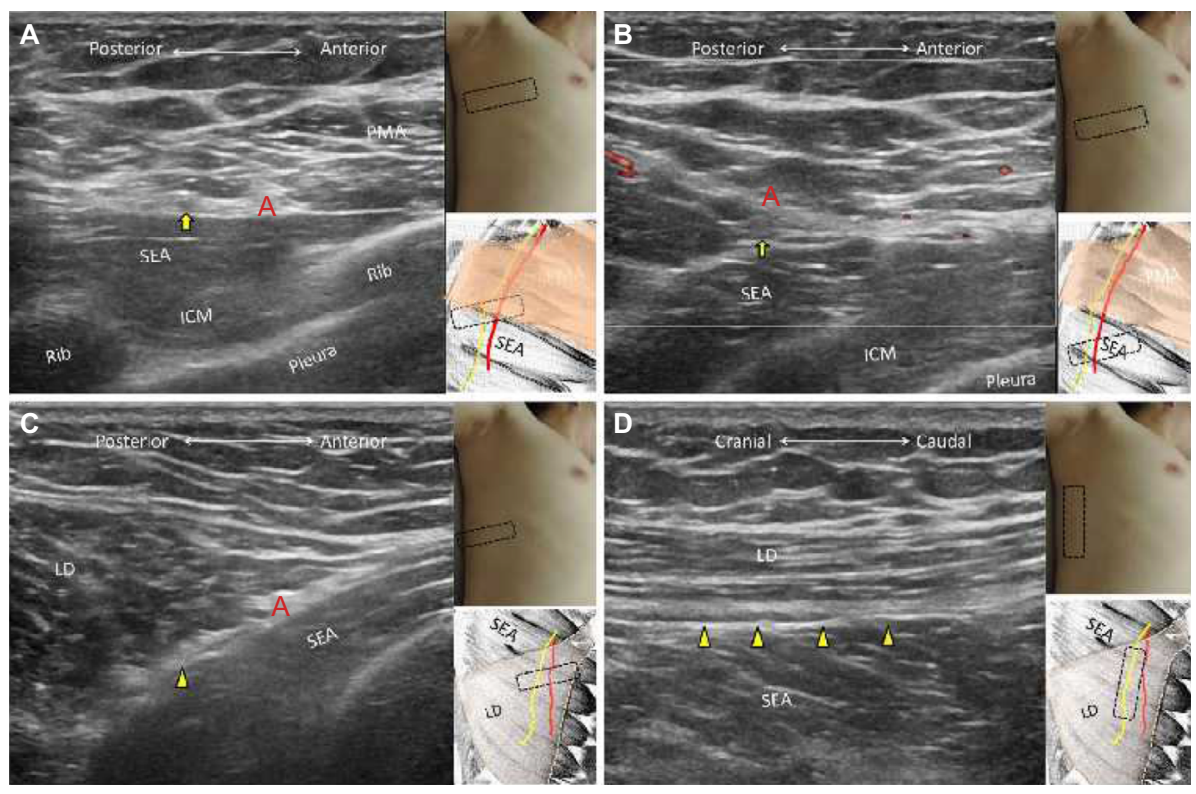

Figure 2 The short axis view of the long thoracic nerve at the junction between pectoralis major and serratus anterior muscles (A) and overlying the serratus anterior muscle (B); the short axis (C) and long axis (D) views of the thoracodorsal nerve.

Notes: Yellow arrows indicate long thoracic nerve; yellow arrowheads indicate thoracodorsal nerve. The red $A$ in $(\mathbf{A})$ and (B) indicates lateral thoracic artery and thoracodorsal artery in (C).

Abbreviations: PMA, pectoralis major; LD, latissimus dorsi; SEA, serratus anterior; ICM, intercostal muscle.

\section{Thoracodorsal nerve}

\section{Anatomy}

The thoracodorsal nerve originates from the posterior cord of the brachial plexus with its fibers coming from the sixth to eighth cervical nerve roots. The nerve descends along the posterior lateral thoracic wall inside the fascia plane between the latissimus dorsi and serratus anterior muscles. The nerve is accompanied by the thoracodorsal artery, a branch of the subscapular artery, and innervates the latissimus dorsi muscle. ${ }^{12}$

\section{Technique}

The arm is abducted and the transducer placed horizontally on the posterior axillary fold. The thoracodorsal nerve courses posterior to the long thoracic nerve and lies in the plane interposed by the latissimus dorsi and serratus anterior muscles. The power Doppler mode can be used to search for the thoracodorsal artery next to which the nerve is seen as a hypoechoic ovoid structure in its short axis (Figure 2C and Video S3). The long axis of the nerve can be depicted as a hypoechoic linear structure between the latissimus dorsi and serratus anterior muscles (Figure 2D).

\section{Lateral and medial pectoral nerves Anatomy}

The lateral pectoral nerve arises from the lateral cord of the brachial plexus with its fibers coming from the fifth to seventh cervical nerve roots. The nerve courses medial to the lateral cord of the brachial plexus and descends in the fascia plane between the pectoralis major and pectoralis minor muscles. It is accompanied by the pectoral branch of the thoracoacromial artery and innervates the pectoralis major muscle. ${ }^{13}$

The medial pectoral nerve arises from the medial cord of the brachial plexus with its fibers coming from the eighth cervical and first thoracic nerve roots. The nerve first descends beneath the pectoralis minor muscle and then pierces it to reach the deep surface of the pectoralis major muscle. It innervates the pectoralis minor muscle and the sternal and costal parts of the pectoralis major muscle. The nerve also receives some communicating fibers from the lateral pectoral nerve in front of the axillary artery. ${ }^{13}$

\section{Technique}

The transducer is placed medial to the coracoid process in the transverse plane to search for the thoracoacromial artery, which is branched from the infraclavicular segment of the axillary artery. The thoracoacromial artery gives off the pectoral, acromial, deltoid, and clavicular branches. The pectoral branch descends between the pectoralis major and pectoralis minor muscles, and the lateral pectoral nerve is usually seen as a hypoechoic ovoid structure accompanying the pectoral branch (Figure 3A and B and Video S4). The medial pectoral nerve branches from the medial cord (Figure 3C) and courses underneath the pectoralis minor muscle (Figure 3D and Video S5). 

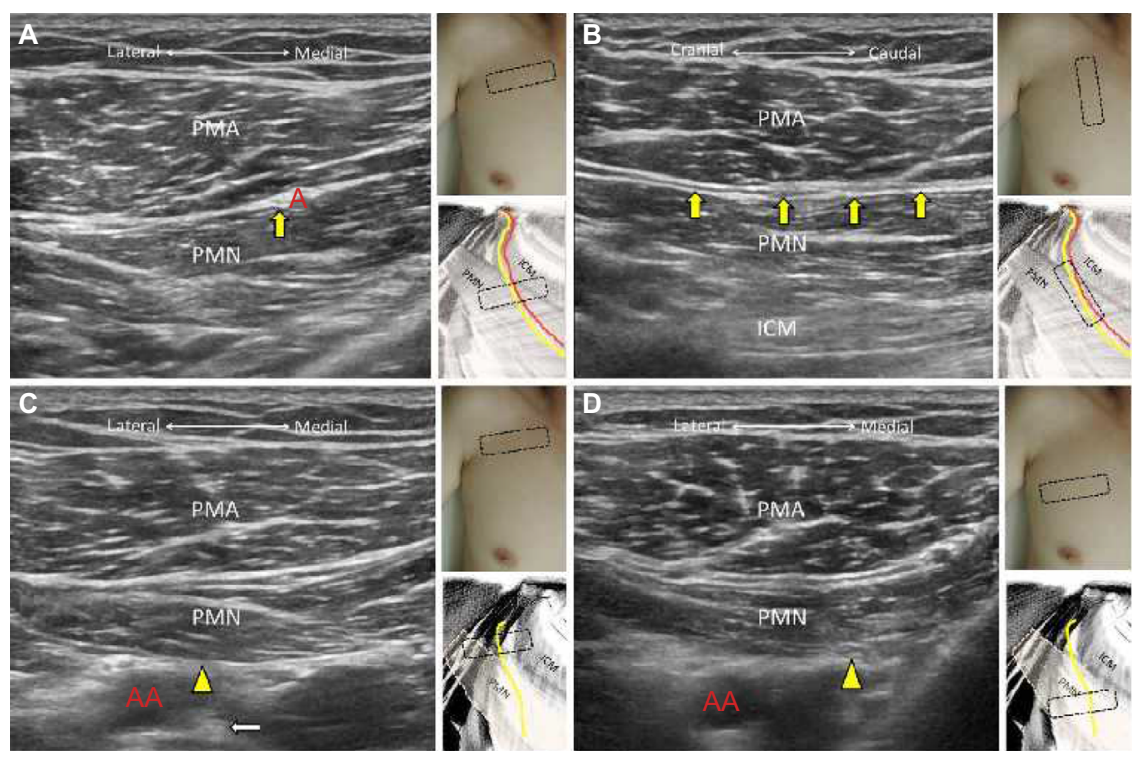

Figure 3 The short axis $(\mathbf{A})$ and long axis $(\mathbf{B})$ views of the lateral pectoral nerve; the short axis views $(\mathbf{C})$ of the medial pectoral nerve at its origin near the medial cord and descending beneath the pectoralis minor muscle (D).

Notes: Yellow arrows indicate the lateral pectoral nerve; yellow arrowheads indicate medial pectoral nerve; white arrow indicates medial cord; red A indicates pectoral branch of the thoracoacromial artery; red AA indicates axillary artery.

Abbreviations: PMA, pectoralis major; PMN, pectoralis minor; ICM, intercostal muscle.

\section{Dorsal scapular nerve Anatomy}

The dorsal scapular nerve originates from the fifth cervical nerve root and then penetrates the medial scalene muscle to run beneath the levator scapulae muscle. After the nerve reaches the interscapular region, it courses under the rhomboid minor and rhomboid major muscles, and above the ribs and intercostal muscles. The nerve is accompanied by the dorsal scapular artery and innervates the levator scapulae, rhomboid minor, and rhomboid major muscles. ${ }^{3,6}$

\section{Technique}

The transducer is placed medial to the superior border of the scapula in the transverse plane. The levator scapulae can be seen attached to the scapular border in its short axis view. The dorsal scapular artery can be found under the power Doppler mode beneath the levator scapulae muscle, and the dorsal scapular nerve is identified as a hypoechoic ovoid structure medial to it. Moving the transducer caudally allows visualization of the dorsal scapular nerve coursing beneath the rhomboid minor and major muscles (Figure 4A and Video S6). Redirecting the transducer in the sagittal plane, the nerve can be seen as a linear hypoechoic structure running above the ribs and intercostal muscles (Figure 4B).

\section{Thoracic and lumbar spinal nerves}

Thoracic $(\mathrm{N}=12)$ and lumbar $(\mathrm{N}=5)$ spines comprise the vertebral body, neural arch, and bony processes. Compared with the thoracic spine, there are no ribs connected to the
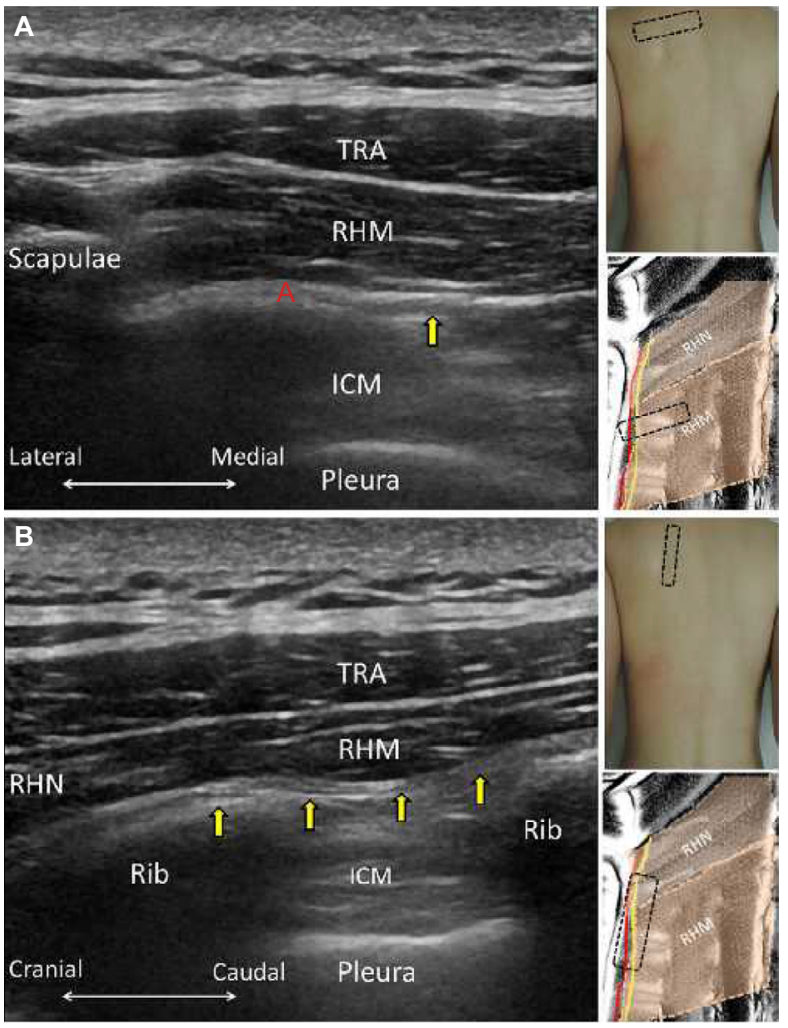

Figure 4 The short axis (A) and long axis (B) views of the dorsal scapular nerve (yellow arrows).

Note: The red A indicates dorsal scapular artery.

Abbreviations: TRA, trapezius; RHM, rhomboid major; RHN, rhomboid minor; ICM, intercostal muscle.

lumbar spine. The intervertebral discs connect the adjacent vertebral bodies, which accompany the facet joints to transit the vertical loading of the vertebral column. The bony processes for each vertebra consist of one spinous process, two 
superior articular processes, two inferior articular processes, and two transverse processes. The neural arch comprises two pedicles, two laminae, and bony processes, as described earlier, encircling the thecal sac. The intervertebral foramen is an orifice bordered by two contiguous vertebral bodies anteriorly, the neural arches superiorly and inferiorly, and the articular processes posteriorly. It allows the exit of the spinal nerves, which are further branched to the ventral and dorsal ramus. Regarding the thoracic spinal nerves, the ventral rami only innervate a narrow segment of muscles and skin over the chest and abdominal wall. In contrast, the ventral rami of the lumbar spinal nerves form plexuses to innervate the pelvic girdles and lower limbs. The dorsal rami are divided into the lateral, intermediate, and medial branches, of which the former two mainly innervate paraspinal muscles and the latter one provides innervation to the facet joints. ${ }^{14,15}$

There are some differences in the configurations between the thoracic and lumbar spine. First, laminae of the inferior vertebrae are superimposed by that of the superior vertebrae in the thoracic spine but not in the lumbar spine, which results in an opening toward the dorsal aspect namely the interlaminar space in the lumbar region. Second, the facet joint planes of the upper thoracic spine are vertically aligned and face backward, whereas those of the lower thoracic spine are still vertically oriented but gradually face toward the midline, which is similar to the pattern of the lumbar spine. Third, the transverse processes of the thoracic spine originate posterior to the articular processes and have connection with the ribs. However, the transverse processes of the lumbar spine lie anterior to the articular process and are directed posterior and lateral. ${ }^{14,15}$

\section{Technique}

\section{Thoracic spinal nerve}

Precise recognition of the numeric numbers of the ribs is helpful for identifying the correct level of the thoracic spine. The transducer is first placed in the sagittal plane on the supraclavicular region near the middle one-third of the clavicle. The first rib is seen as a hyperechoic linear structure above the subclavian artery and brachial plexus. In the sagittal view, the bony reflection of the first rib under sonography is longer than the remaining ribs, because it allows visualization of the lateral angled portion, which is more parallel to the scanning plane of the transducer (Figure 5A and Video S7). The transducer is gradually relocated to the midline along the paraspinal line and the ribs (round and hyperechoic), which are intercepted by intercostal spaces, containing the pleura and costotransverse ligaments. Between the pleura and costotransverse ligament is the thoracic paravertebral space, medial to which are the exits of the thoracic spinal nerves. Moving the transducer more medially until it crosses the costotransverse joints, the dorsal surface of the transverse processes and articular processes are visualized (Figure 5B), followed by the lamina (Figure 5C) and spinous processes (Figure 5D and Video S8). Since the laminae of the thoracic spine are overlapped, it is difficult to visualize the interlaminar space, especially at the upper thoracic regions. The transducer can be redirected to scan the thoracic spine in the short axis view and to identify the spinous processes, laminae, articular processes, costotransverse joints, and ribs from the midline to lateral paraspinal line (Figure 5E). Slight relocation of the transducer between both ribs enables depiction of the pleura and the paravertebral space in the transverse view (Figure 5F and Video $\mathrm{S} 9$ ).

\section{Lumbar region}

The transducer is first placed in the midline between the two posterior superior iliac spines for scanning the short axis view of the lumbar spine. The dorsal surface of the sacrum appears as a dual-sloped roof, the ridge of which is the medial sacral crest (Figure 6A). The transducer is relocated more cranially until a gap is seen in the middle of the roof, indicating the appearance of the L5/S1 interlaminar space. The lateral border of the L5/S1 interlaminar space comprises bilateral L5 inferior articular processes and S1 superior articular processes. The ala of the sacrum is visualized as a hyperechoic bony plane extending from the base of the S1 superior articular process, and the medial branch of L5 spinal nerve courses around the corner formed by both bony elements (Figure 6B).

The spinous process and lamina of L5 are seen after passing the L5/S1 interlaminar space. Moving the transducer more cranially allows visualization of the L4/L5 facet joint and the L5 transverse process (Figure 6C). The L4 spinal nerve is anticipated exiting from the intervertebral foramen deep to the intertransversarii lumborum, a small muscle placed connecting the transverse processes of adjacent vertebrae (Figure 6D and Video S10). The L4 medial branch is supposed to be present at the intersection of the base of L5 superior articular process and L5 transverse process. The L1-L3 spinal nerves and their medial branch can be scanned in the similar manner.

When scanning the lumbar spine in the sagittal plane, the transducer can be placed in the midline to see the spinous processes (hyperechoic round lumps) connected by the supraspinous and interspinous ligaments (Figure 7A). More laterally, the laminae are arranged like "horse heads", and the ligamentum flavum, dura, thecal cord, and 

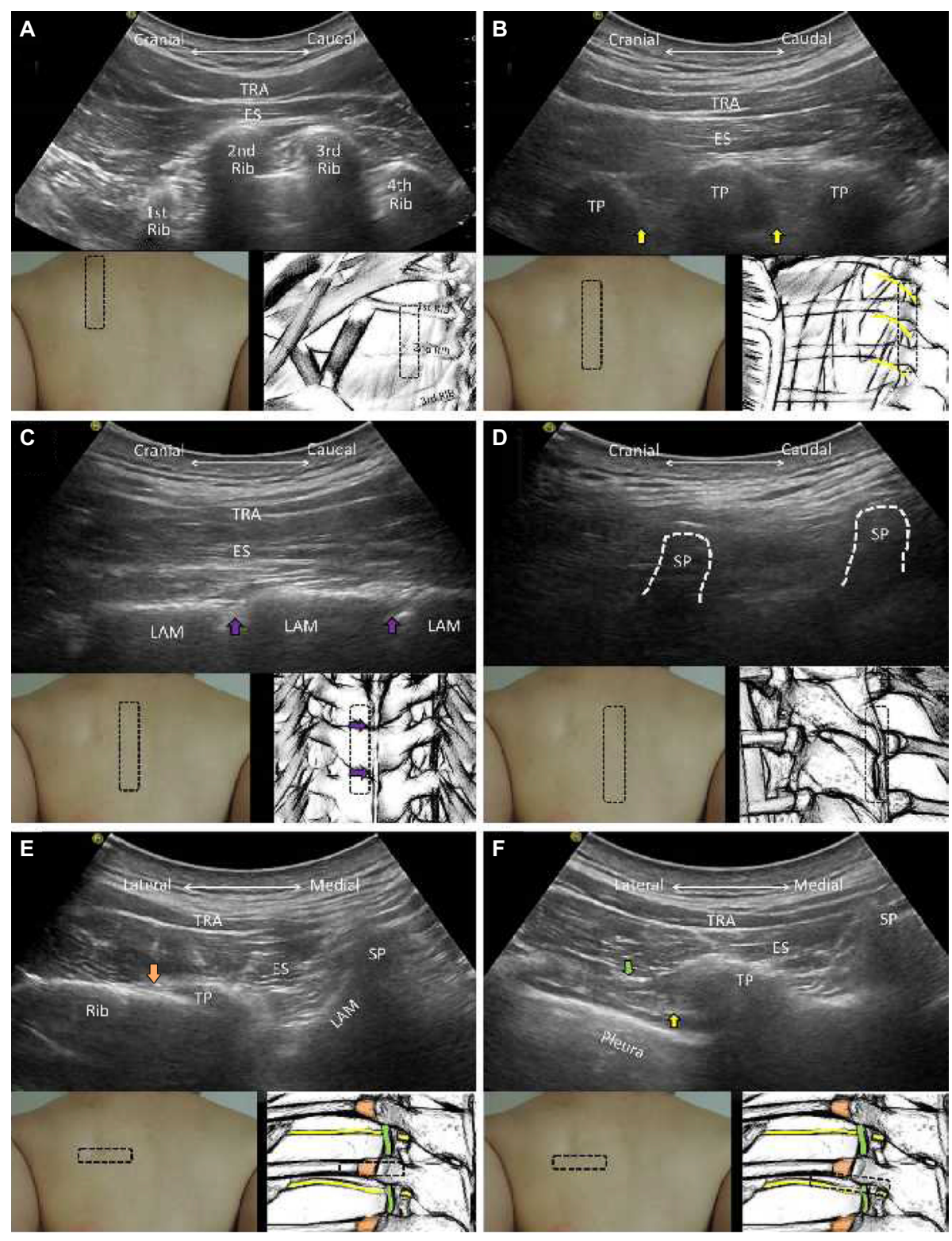

Figure 5 The sagittal planes for the ribs (A), transverse processes (B), laminae (C), and spinous processes of the thoracic vertebrae (D). The horizontal planes for the transverse processes $(\mathbf{E})$ and the intercostal space $(\mathbf{F})$ of the thoracic vertebrae.

Notes: Yellow arrows indicate spinal nerve roots; purple arrows indicate interlaminar spaces; orange arrows indicate lateral costal transverse ligament; green arrows indicate superior costal transverse ligament.

Abbreviations: TRA, trapezius; ES, erector spinae; TP, transverse process; SP, spinous process; LAM, lamina.

anterior border of the vertebral canals can be seen through the interlaminar space (Figure 7B). Moving the transducer further laterally, the articular processes of different vertebrae are adjoined by facet joints, which are shaped like "camel humps" (Figure 7C), and the nerve roots are seen exiting from the intervertebral foramina caudal to the superior articular processes (Figure 7D). More laterally, the transverse processes are seen in their short axis view, looking like a trident. The lumbar plexus is located in the plane between the intertransversarii lumborum superficially and psoas major deeply (Figure 7E and Video S11). The transducer can be relocated to the posterior and lateral aspect of the lumbar region to visualize the oblique sagittal view of the lumbar plexus (Figure 7F).

\section{Intercostal nerve}

\section{Anatomy}

The intercostal nerves originate from the anterior rami of the thoracic spinal nerves. The intercostal nerves of T3-T6 only innervate the thoracic wall. However, in addition to innervating the thoracic cavity, intercostal nerves of $\mathrm{T} 1$ and T2 join the brachial plexus to provide cutaneous sensation of the medial arm. Those of T7-T11 supply the abdomen and peritoneum. The nerves course anteriorly in the plane 

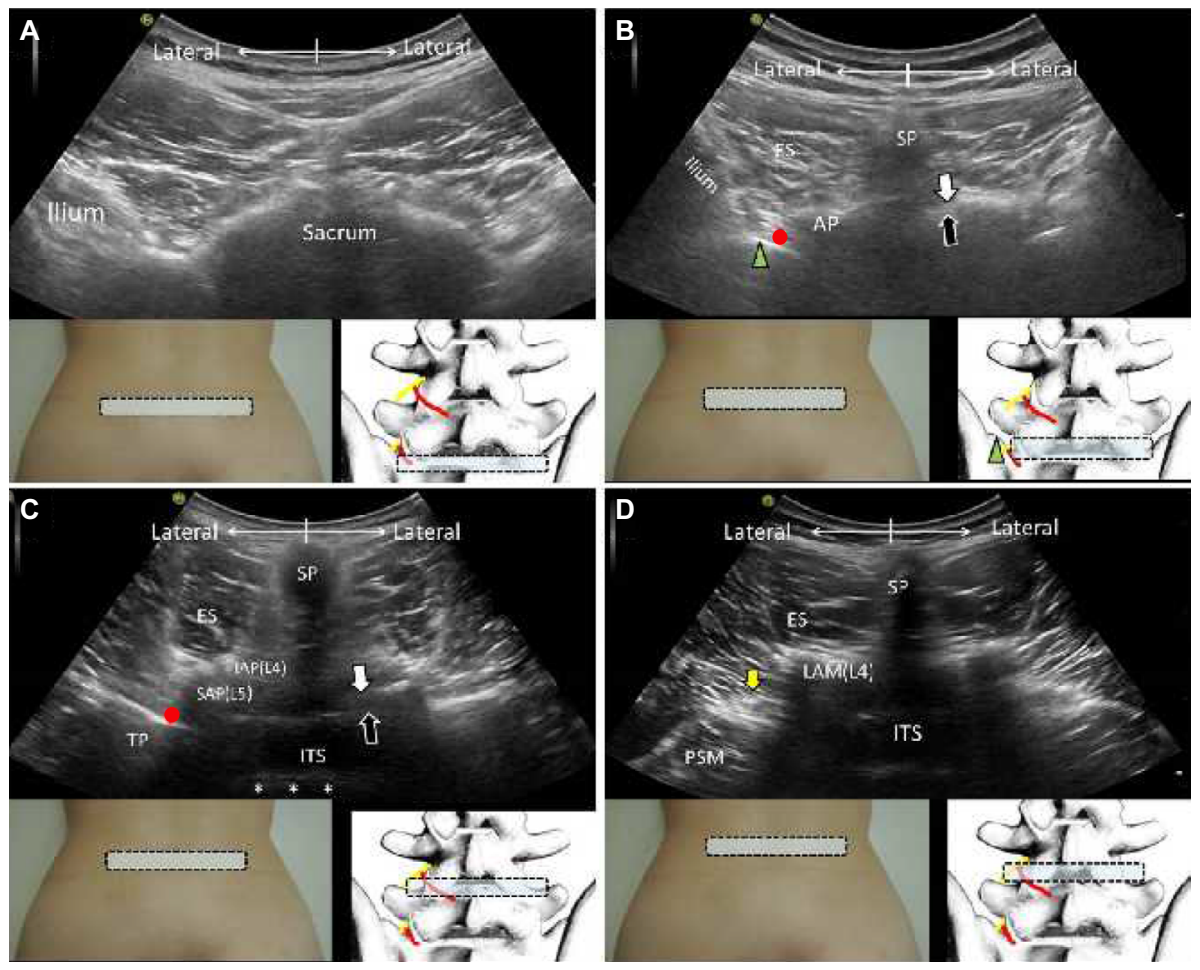

Figure 6 The horizontal planes for the cranial sacrum (A), alae of the sacrum (B), bilateral L4/L5 facet joints (C), and waist of the L4 lamina (D).

Notes: Green arrowheads indicate ala of the sacrum; red circles indicate medial branch; yellow arrows indicate nerve roots; white arrows indicate the ligamentum flavum; black arrows indicate the dura. The asterisks indicate the posterior longitudinal ligament.

Abbreviations: ES, erector spinae; AP, articular process; SP, spinous process; TP, transverse process; SAP, superior articular process; IAP, inferior articular process; ITS, intrathecal space; LAM, lamina; PSM, psoas major.

between the internal and innermost intercostal muscles and under the lower edge of the ribs. ${ }^{16}$

\section{Technique}

The patient lies prone and abducts the arm to expose the whole thoracic wall. The transducer is placed perpendicular to two consecutive ribs. Three layers of the intercostal muscles can be identified connecting them. The pleura, appearing as a hyperechoic linear structure, can be seen deep to the intercostal muscles and slides during respiration (Figure 8A). Gradually relocating the transducer laterally allows the visualization of the intercostal nerves, along with intercostal vessels, in the short axis view in the plane between the innermost and internal intercostal muscles and caudal to the lower edge of the rib above (Figure 8B and Video S12).

\section{Iliohypogastric and ilioinguinal nerves \\ Anatomy}

The iliohypogastric and ilioinguinal nerves are both branches of the first lumbar spinal nerves. They arise from the lateral border of the psoas major muscle and descend laterally across the quadratus lumborum and iliacus muscles. The iliohypogastric nerve runs parallel and cranial to the ilioinguinal nerve along the lateral abdominal wall. Near the anterior superior iliac spine, both nerves pierce the transverse abdominis muscle and course in the plane interposed by the transverse abdominis and internal oblique muscles. ${ }^{17}$

The iliohypogastric nerve has two branches. The lateral cutaneous branch courses through the internal and external oblique muscles above the iliac crest and then provides cutaneous sensation in the gluteal region. The anterior cutaneous branch penetrates the internal and external oblique muscles above the opening of the inguinal ring and innervates the hypogastric region. ${ }^{17}$

The ilioinguinal nerve pierces the internal oblique muscle and then runs with the spermatid cord though the superficial inguinal ring, providing cutaneous sensation at the proximal and medial thigh. ${ }^{17}$

\section{Technique}

The transducer is placed at the level of the anterior superior iliac spine and is pivoted to the direction perpendicular to the inguinal ligament. Three layers of the abdominal muscles are attached to the iliac crest. Ilioinguinal and 

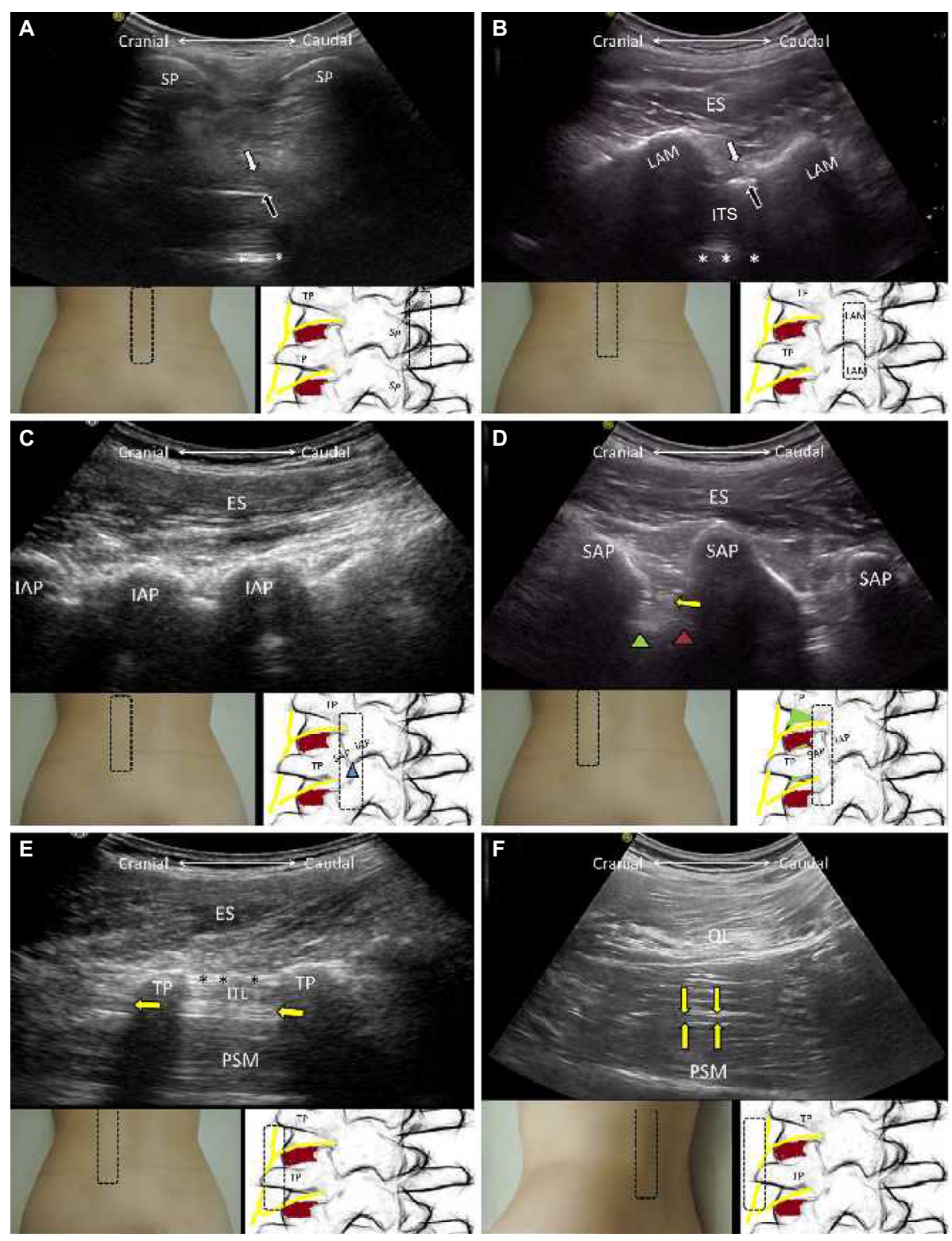

Figure 7 The sagittal planes for the spinous processes (A), laminae (B), inferior articular processes (C), superior articular processes (D), and transverse processes (E) of the lumbar spine; the oblique sagittal plane for the lumbar plexus $(\mathbf{F})$.

Notes: Green arrowheads indicate vertebral bodies; brown arrowheads indicate intervertebral discs; yellow arrows indicate nerve roots or lumbar plexus; black asterisks indicate intertransverse ligaments; white asterisks indicate anterior complex containing the posterior longitudinal ligament and anterior dura.

Abbreviations: ES, erector spinae; SP, spinous process; TP, transverse process; SAP, superior articular process; IAP, inferior articular process; ITS, intrathecal space; LAM, lamina; PSM, psoas major; QL, quadratus lumborum; ITL, intertransversalis lumborum.

iliohypogastric nerves appear as two hypoechoic ovoid structures in the plane between internal oblique and transverse abdominis muscles adjacent to the iliac crest. The ilioinguinal nerve is located lateral to the iliohypogastric nerve at the level of anterior superior iliac spine (Figure 9A). If a hypoechoic round structure is seen in the same plane but away from the iliac crest, it is the subcostal nerve (12th thoracic spinal nerve). While scanning those nerves, the power Doppler mode can be used to locate the deep circumflex iliac artery, which is situated in the same fascia plane as the nerves described earlier (Figure 9B and Video S13).

\section{Superior cluneal nerve Anatomy}

The superior cluneal nerve originates from the dorsal rami of the spinal nerves (T11-L4) and is divided into the medial, intermediate, and lateral branches. The nerve passes through the psoas major and paraspinal muscles and courses caudally until reaching the iliac crest. It then pierces the thoracolumbar 


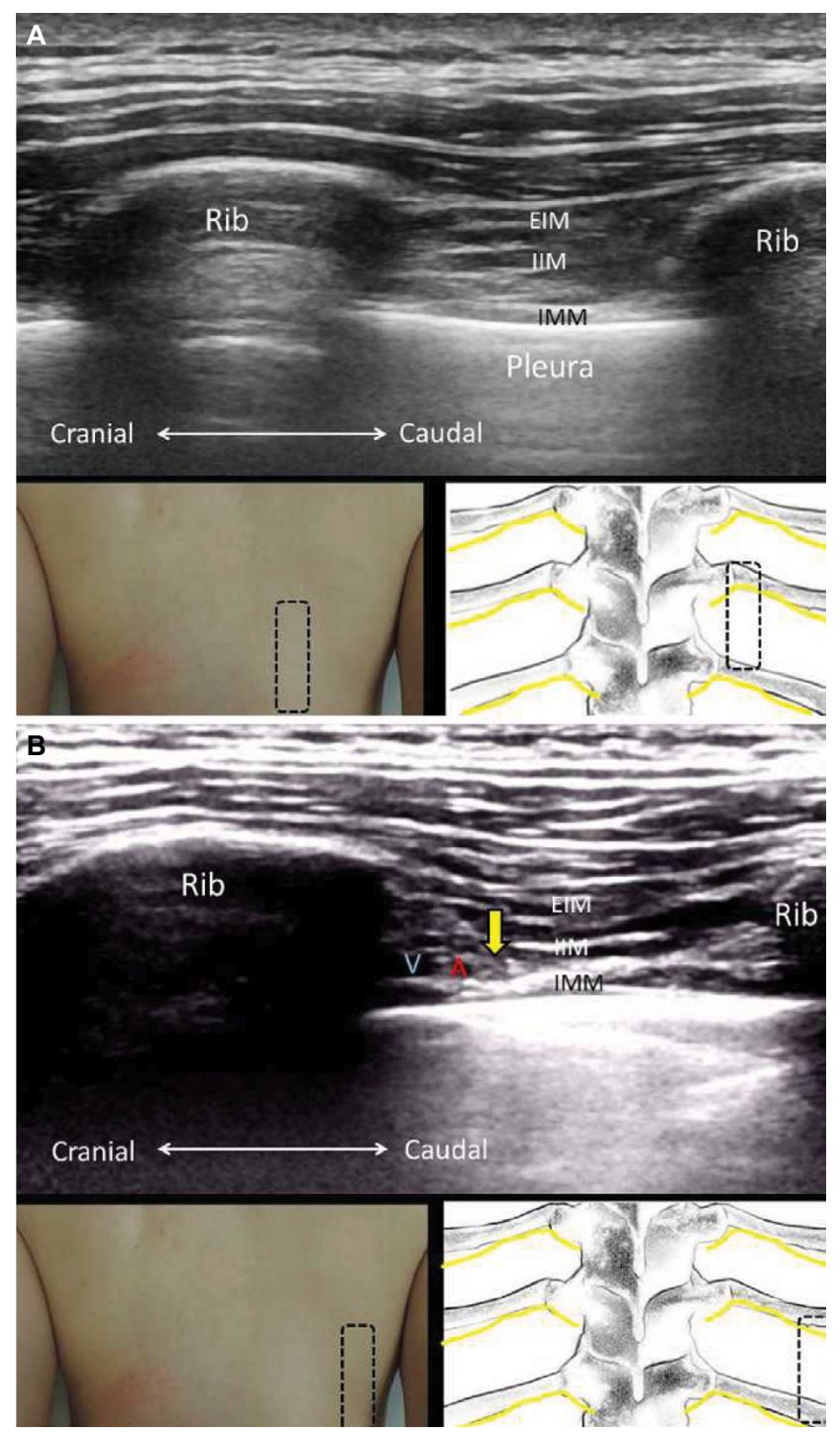

Figure 8 The sagittal plane for the origins $(\mathbf{A})$ and middle portions $(\mathbf{B})$ of the ribs. Notes: Yellow arrow indicates intercostal nerve; the red $A$ indicates intercostal artery.

Abbreviations: EIM, external intercostal muscle; IIM, internal intercostal muscle; IMM, innermost intercostal muscle; $\mathrm{V}$, intercostal vein.

fascia and provides cutaneous sensation of the gluteal region. Different from the other two branches, the medial branch runs through an osteofibrous tunnel, formed by the thoracolumbar fascia superficially and the iliac crest deeply. ${ }^{18}$

\section{Technique}

The transducer is placed in the transverse plane near the posterior inferior iliac spine and is slowly moved along the medial edge of the iliac crest. The most superficial muscle, the gluteus maximus, becomes gradually thinner until it disappears with the emergence of the gluteus medius muscle. The superior cluneal nerve, appearing as a hypoechoic round structure in its short axis view, is seen between the thoracolumbar fascia and the iliac crest (Figure 10A and Video S14). Redirecting
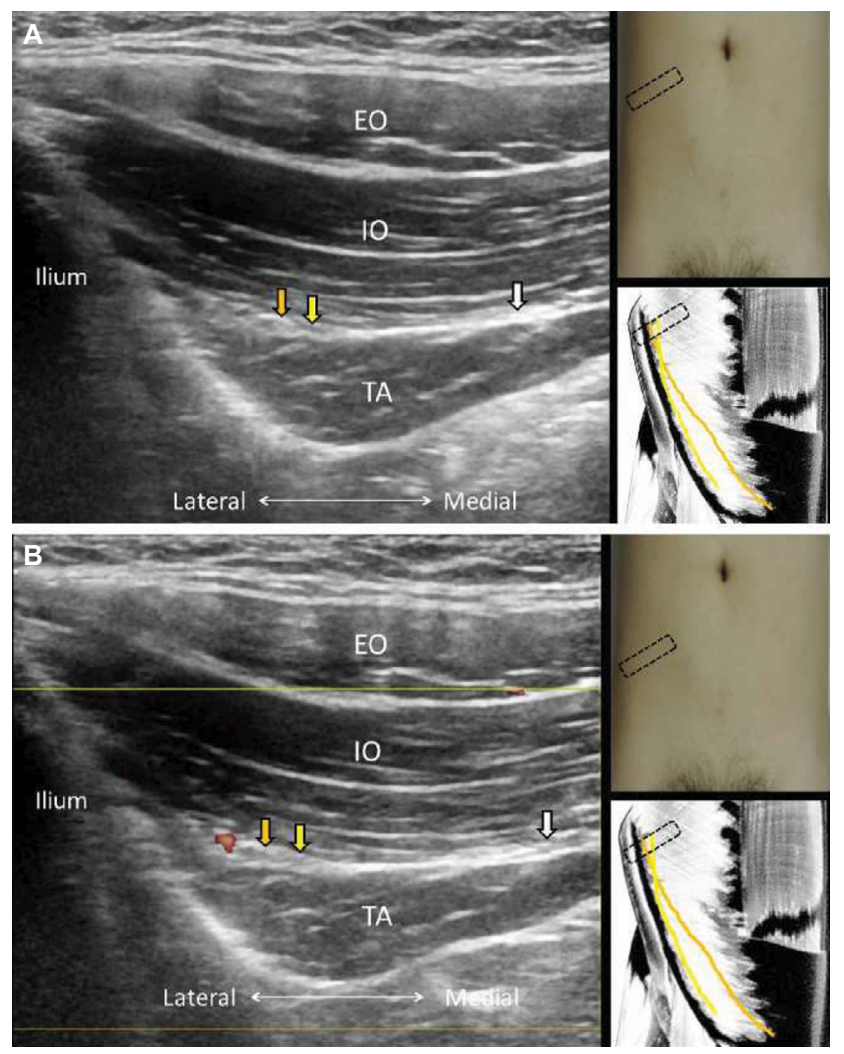

Figure 9 Gray scale (A) and power Doppler imaging (B) for the ilioinguinal, iliohypogastric, and subcostal nerves.

Notes: Orange arrows indicate ilioinguinal nerve; yellow arrows indicate iliohypogastric nerve; white arrows indicate subcostal nerve.

Abbreviations: EO, external oblique muscle; IO, internal oblique muscle; TA, transverse abdominis muscle.

the transducer in the sagittal plane allows visualization of the nerve coursing above the gluteus medius muscle (Figure 10B).

\section{Conclusion}

Clear recognition of trunk neuroanatomy by using highresolution US is essential for pain management and related research. Scanning peripheral nerves requires robust background of three-dimensional human anatomy. In the trunk region, tracing the full course of certain nerves may be challenging because some are deeply situated or hindered by the bony structures such as clavicles, ribs, and vertebrae. Interchange between the horizontal and sagittal planes is sometimes required to localize the correct spinal level. Familiarization with the osseous characteristics of the vertebrae is helpful in clarifying paraspinal sonoanatomy and further displaying spinal nerves and relevant branches.

\section{Disclosure}

The current research was supported by Ministry of Science and Technology, Taiwan, Republic of China (MOST 105-2314-B-002-008). The authors report no other conflicts of interest in this work. 

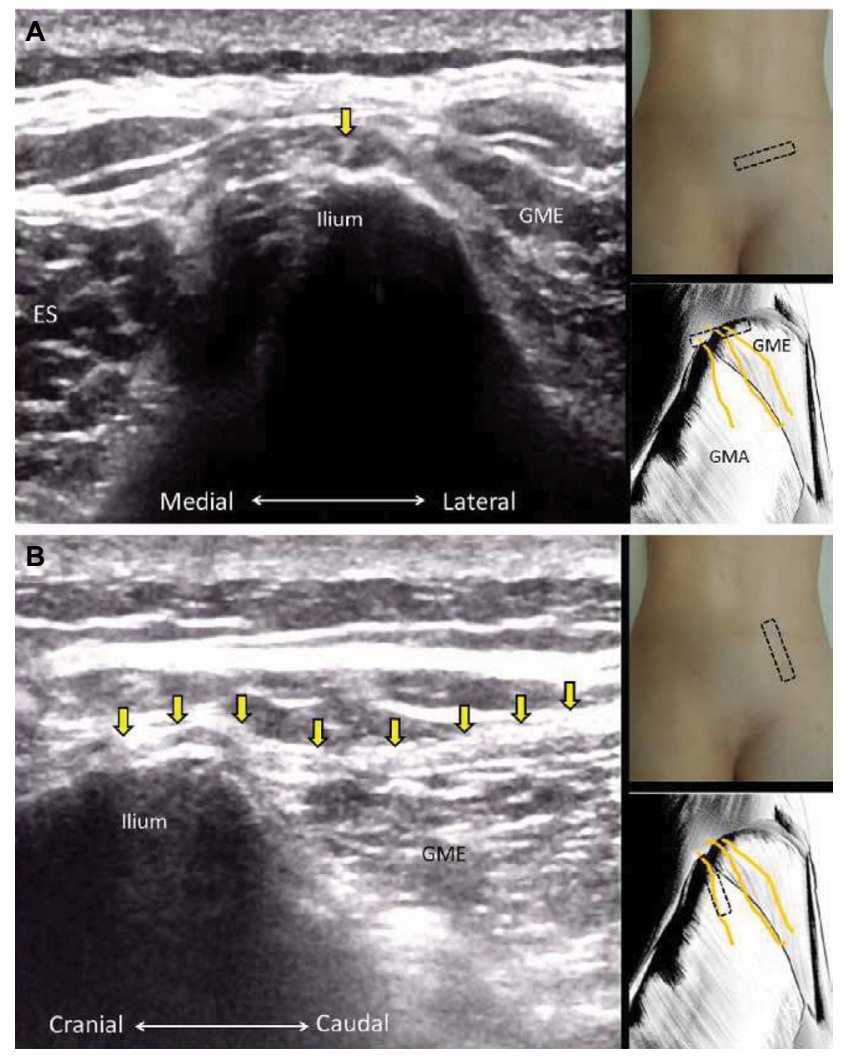

Figure 10 The short axis (A) and long axis (B) views of the superior cluneal nerve. Note: Yellow arrows indicate superior cluneal nerve.

Abbreviations: GME, gluteus medius; ES, erector spinae.

\section{References}

1. Wu CH, Chang KV, Ozcakar L, et al. Sonographic tracking of the upper limb peripheral nerves: a pictorial essay and video demonstration. $\mathrm{Am}$ J Phys Med Rehabil. 2015;94(9):740-747.

2. Hung CY, Hsiao MY, Ozcakar L, et al. Sonographic tracking of the lower limb peripheral nerves: a pictorial essay and video demonstration. $\mathrm{Am}$ J Phys Med Rehabil. 2016;95(9):698-708.

3. Chang KV, Lin CP, Hung CY, Ozcakar L, Wang TG, Chen WS. Sonographic nerve tracking in the cervical region: a pictorial essay and video demonstration. Am J Phys. Med Rehabil. Epub June 26, 2016.
4. Demondion X, Herbinet P, Van Sint Jan S, Boutry N, Chantelot C, Cotten A. Imaging assessment of thoracic outlet syndrome. RadioGraphics. 2006;26(6):1735-1750.

5. Martinoli C, Gandolfo N, Perez MM, et al. Brachial plexus and nerves about the shoulder. Semin Musculoskelet Radiol. 2010;14(5): 523-546.

6. Sultan HE, Younis El-Tantawi GA. Role of dorsal scapular nerve entrapment in unilateral interscapular pain. Arch Phys Med Rehabil. 2013;94(6):1118-1125.

7. Greher M, Moriggl B, Peng PW, Minella CE, Zacchino M, Eichenberger U. Ultrasound-guided approach for L5 dorsal ramus block and fluoroscopic evaluation in unpreselected cadavers. Reg Anesth Pain Med. 2015;40(6):713-717.

8. Karkucak M, Kurt M, Ozcakar L. Ultrasound video demonstration for lumbar facet joint injection. Am J Phys Med Rehabil. 2016;95(10): e165-166.

9. Sala-Blanch X, Reina MA, Pangthipampai P, Karmakar MK. Anatomic basis for brachial plexus block at the costoclavicular space: a cadaver anatomic study. Reg Anesth Pain Med. 2016;41(3):387-391.

10. Lapegue F, Faruch-Bilfeld M, Demondion X, et al. Ultrasonography of the brachial plexus, normal appearance and practical applications. Diagn Interv Imaging. 2014;95(3):259-275.

11. Nasu H, Yamaguchi K, Nimura A, Akita K. An anatomic study of structure and innervation of the serratus anterior muscle. Surg Radiol Anat. 2012;34(10):921-928.

12. Takahashi N, Watanabe K, Koga N, et al. Anatomical research of the three-dimensional route of the thoracodorsal nerve, artery, and veins in latissimus dorsi muscle. Plast Reconstr Surg. 2013;1(2):1-7.

13. $\mathrm{Kg} \mathrm{P}, \mathrm{K} \mathrm{S}$. Anatomical study of pectoral nerves and its implications in surgery. J Clin Diagn Res. 2014;8(7):AC01-AC05.

14. Cho JC, Haun DW, Kettner NW. Sonographic evaluation of the greater occipital nerve in unilateral occipital neuralgia. J Ultrasound Med. 2012;31(1):37-42.

15. Provenzano DA, Narouze S. Sonographically guided lumbar spine procedures. J Ultrasound Med. 2013;32(7):1109-1116.

16. Bhatia A, Gofeld M, Ganapathy S, Hanlon J, Johnson M. Comparison of anatomic landmarks and ultrasound guidance for intercostal nerve injections in cadavers. Reg Anesth Pain Med. 2013;38(6):503-507.

17. Tagliafico A, Bignotti B, Cadoni A, Perez MM, Martinoli C. Anatomical study of the iliohypogastric, ilioinguinal, and genitofemoral nerves using high-resolution ultrasound. Muscle Nerve. 2015;51(1): $42-48$.

18. Bodner G, Platzgummer H, Meng S, Brugger PC, Gruber GM, Lieba-Samal D. Successful identification and assessment of the superior cluneal nerves with high-resolution sonography. Pain physician. 2016; 19(3):197-202.

\section{Journal of Pain Research}

\section{Publish your work in this journal}

The Journal of Pain Research is an international, peer reviewed, open access, online journal that welcomes laboratory and clinical findings in the fields of pain research and the prevention and management of pain. Original research, reviews, symposium reports, hypothesis formation and commentaries are all considered for publication.

\section{Dovepress}

The manuscript management system is completely online and includes a very quick and fair peer-review system, which is all easy to use. Visit http://www.dovepress.com/testimonials.php to read real quotes from published authors. 